Pacific Journal of Mathematics

CHARACTERIZATIONS OF DIRECT SUMS AND COMMUTING 


\title{
CHARACTERIZATIONS OF DIRECT SUMS AND COMMUTING SETS OF VOLTERRA OPERATORS
}

\author{
G. K. KALISCH
}

Theorem 1 contains an abstract characterization and unitary invariants of operators $T$ which are finite direct sums of $n$ Volterra operators $\left(\alpha_{j} V f\right)(x)=\alpha_{j} \int_{0}^{x} f(y) d y$ with real nonzero $\alpha_{j}$ defined on a Hilbert space $\mathscr{H}$ which is a direct sum of $n$ $\mathscr{L}_{2}\left(I_{1}\right)$ spaces on the unit interval $I_{1}$. This is done by demanding that the dimension of $\left(T+T^{*}\right) \mathscr{H}$ be $n$; that the subspaces $\mathscr{K}_{j}$ of $\mathscr{H}$ generated by $T$ and the eigenvectors $e_{j}$ of $T+T^{*}$ be orthogonal to all $e_{k}$ for $k \neq j$; and that the spectrum of $T$ be 0 . Theorem 2 contains an abstract characterization and unitary invariants of finite commuting sets $\left\{W_{j}\right\}_{1}^{n}$ of Volterra operators which are real nonzero multiples of integration in the various coordinate axis directions on a Hilbert space $\mathscr{H}$ which is the $\mathscr{L}_{2}$ space on the unit cube in $n$ real dimensions. The characterization is given by demanding that the $W_{j}$ commute with all $W_{k}$ and $W_{k}^{*}$ for $k \neq j$; that $\Pi\left(W_{j}+W_{j}^{*}\right) \mathscr{H}=\mathscr{E}$ have dimension 1 ; that $\mathscr{H}$ be spanned by the $W_{j}$ 's and $\mathscr{E}$; and that the $W_{j}$ 's have spectrum 0 .

The simplest bounded Hermitian operators are the simple or cyclic operators which are defined as "multiplication by the independent variable on a suitable $\mathscr{L}_{2}(\mu)$ space" where $\mu$ is a Borel measure with compact support on the real line. The simplest bounded Volterra operators are of the form $\alpha V$ for real nonzero $\alpha$ defined on $\mathscr{L}_{2}\left(I_{1}\right)$. In general, we mean by Volterra operator a linear transformation $T_{F}$ defined on a space of functions so that $\left(T_{F} f\right)(x)=\int_{0}^{x} F(x, y) f(y) d y$. It is a remarkable fact that the simple Hermitian operators depend unitarily on the measure $\mu$, that is, two such operators are unitarily equivalent if and only if the corresponding measures $\mu$ are equivalent, while two Volterra operators $V$ defined on different $\mathscr{L}_{2}(\mu)$ spaces are unitarily equivalent so long as both measures $\mu$ are nonatomic and have the same total mass. Thus there is no loss of generality in our paper if we confine ourselves to the Lebesgue spaces $\mathscr{L}_{2}\left(I_{1}\right)$, etc. The presence of atoms brings our different phenomena which we hope to develop in the future.

The most general Hermitian operators are direct sums of the simple ones. This motivates our aim to characterize direct sums of real multiples of the simplest Volterra operators. This work follows the spirit of [3] in that we seek to establish concrete analytic representations. Our Theorem 1 is a first steps in this direction. While these 
operators fall into the class of operators considered in Livšic's theory $[1,4]$, the conclusions of our theorem have to be deduced from that theory in approximately the same way we proceed, namely by basing it upon the characterization of $V$ itself [3].

Another representation theorem for Hermitian operators says that commuting families can be simultaneously represented as families of multiplication operators by functions on some suitable $\mathscr{L}_{2}(\mu)$ space with Borel measure $\mu$ of finite mass in $I_{1}$; see for example [3]. This motivates our interest in the corresponding situation for Volterra operators which is explored in our Theorem 2. The proof is again based on the characterization of $V$ itself [3]; in addition it uses a lemma due to Livšic in the form stated on p. 354 of [3] and referred to here as "Livšic's Lemma". The proof then proceeds by establishing explicit formulas for two analytic functions in $n$ and $2 n$ variables respectively determined by products of the resolvents of the $W^{\prime} \mathrm{s}$; one of these functions is the joint characteristic function of the set $\left\{W_{j}\right\}$ [3].

We write $I_{n}$ for the unit cube in the space $R_{n}$ of $n$ real dimensions and $\mathscr{L}_{2}\left(I_{n}\right)$ for the Lebesgue $\mathscr{L}_{2}$ space on $I_{n}$. We define the operator $V_{j}$ on $\mathscr{L}_{2}\left(I_{n}\right)$ as $\left(V_{j} f\right)\left(x_{1}, \cdots, x_{n}\right)=\int_{0}^{x_{j}} f\left(x_{1}, \cdots, \xi_{j}, \cdots, x_{n}\right) d \xi_{j}$. We say that the Hilbert space $\mathscr{H}$ is generated by the set $\left\{T_{j}\right\}$ of operators and the subset $\mathscr{S} \subset \mathscr{H}$ if $\mathscr{H}$ is the least closed subspace of $\mathscr{H}$ containing $\mathscr{S}$ and invariant under each $T_{j}$. We say that the set $\left\{S_{j}\right\}$ of operators on the Hilbert space $\mathscr{H}$ is isomorphic (more precisely isometrically isomorphic) with the set $\left\{T_{j}\right\}$ of operators on the Hilbert space $\mathscr{K}$ if there is an isometry $U$ of $\mathscr{\mathscr { C }}$ onto $\mathscr{K}$ such that $U S_{j}=$ $T_{j} U$ for all $j$.

The following two theorems state easily verified necessary conditions; it is their sufficiency we are concerned with here.

THEOREM 1. Let the operator $T$ be defined on the Hilbert space $\mathscr{H}$ such that

(i ) $\operatorname{dim}\left(T+T^{*}\right) \mathscr{H}=n$ and $\left(T+T^{*}\right) e_{j}=\alpha_{j} e_{j}$ for $j=1, \cdots, n$ where $\left\{e_{j}\right\}$ is orthonormal and the $\alpha_{j}$ are nonzero real numbers;

(ii) if $\mathscr{H}_{j}$ is the subspace of $\mathscr{H}$ generated by $T$ and $e_{j}$ then $\mathscr{H}_{j} \perp e_{k}$ for all distinct $j$ and $k$;

(iii) $\mathscr{H}$ is generated by $T$ and the set $\left\{e_{j}\right\}$;

(iv) the spectrum of $T=0$.

Then $T$ on $\mathscr{H}$ is isomorphic with the direct sum $\sum_{j=1}^{n} \oplus \alpha_{j} V$ defined on the direct sum of $n$ copies of $\mathscr{L}_{2}\left(I_{1}\right)$. Two operators $T$ as above are unitarily equivalent if and only if they have the same $\alpha$ 's as defined in (i) above.

REMARKs. (1) Our demanding that the $\alpha$ 's in (i) be real reflects 
the crucial role that the hypothesis that $\operatorname{dim}\left(V+V^{*}\right) \mathscr{L}_{2}\left(I_{1}\right)=1$ plays in the proof of the representation theorem for $V$ [3]; it is in fact an obvious necessary condition. A trivial extension of our theorem is of course possible if there exists a complex number $\beta$ such that $\beta T$ satisfies condition (i). The case for general complex $\alpha_{j}$ does not fall within the scope of our technique.

(2) Turning now to hypothesis (ii), the example $S=i V^{2}$ shows that $\operatorname{dim}\left(S+S^{*}\right) \mathscr{\mathscr { C }}=\operatorname{dim} \mathscr{E}=2$ while any single nonzero vector $e \in \mathscr{E}$ has the property that the subspace $(S ; e)$ of $\mathscr{C}$ generated by $S$ and $e$ is all of $\mathscr{H}$ (see for example [2], Lemma 7). Thus $S$ is certainly not isomorphic with any operator of the form $\alpha V \oplus \beta V$.

Proof of Theorem 1. We base the proof on the case $n=1$, the characterization of $\alpha V$, found in [3]. We show below that for distinct $j$ and $k$, we have $\mathscr{H}_{j} \perp \mathscr{H}_{k}$; then (iii) implies that $\mathscr{\mathscr { C }}=\sum \oplus \mathscr{H}_{j}$. The definition of $\mathscr{C}_{j}$ implies that $T \mathscr{C}_{j} \subset \mathscr{K}_{j}$ and the mutual orthogonality of the $\mathscr{H}_{j}$ implies that $T^{*} \mathscr{K}_{j} \subset \mathscr{H}_{j}$. Therefore the restriction $T_{j}$ of $T$ to $\mathscr{H}_{j}$ has the property $\left(T_{j}\right)^{*}=\left(T^{*}\right)_{j}$ so that $T_{j}$ satisfies the hypotheses of our theorem for $n=1$ and $T_{j}$ on $\mathscr{C}_{j}$ is isomorphic with $\alpha_{j} V$ on $\mathscr{L}_{2}\left(I_{1}\right)$ by [3]; thus the proof is complete. It remains to show that $\mathscr{X}_{j} \perp \mathscr{C}_{k}$ for distinct $j$ and $k$. Note first that (i) implies that for all $x \in \mathscr{H}$ we have $\left(T+T^{*}\right) x=\sum \alpha_{j}\left(x, e_{j}\right) e_{j}$ and hence (ii) implies that $\left(T+T^{*}\right) T^{n} e_{j}=T^{n+1} e_{j}+T^{*} T^{n} e_{j}=\alpha_{j}\left(T^{n} e_{j}, e_{j}\right) e_{j}$ so that, still by (ii), we have $T^{*} T^{n} e_{j} \in \mathscr{H}_{j}$ for all nonnegative integers $n$, whence $T^{*} \mathscr{H}_{j} \subset \mathscr{H}_{j}$ and $T^{* n} \mathscr{H}_{j} \subset \mathscr{H}_{j}$ for all nonnegative integers $n$. But then (ii) implies that $e_{k} \perp T^{* n} \mathscr{C}_{j}$, i.e., $T^{n} e_{k} \perp \mathscr{H}_{j}$, which implies that $\mathscr{K}_{k} \perp \mathscr{H}_{j}$ as desired. The fact that the set $\left\{\alpha_{j}\right\}$ determines $T$ unitarily is then an immediate consequence of our representation.

THEOREM 2. Let a finite set of $n$ operators $\left\{W_{j}\right\}$ be defined on the Hilbert space $\mathscr{Y}$ such that

(i ) $\left(\prod_{j=1}^{n}\left(W_{j}+W_{j}^{*}\right)\right) \mathscr{H}$ has dimension one and is spanned by the element $e$ of norm one;

(ii) all $W_{j}$ commute with all $W_{k}$ and $W_{k}^{*}$ for all $k \neq j$;

(iii) $\mathscr{H}$ is generated by the set $\left\{W_{j}\right\}$ and $e$;

(iv) the spectrum of every $W_{j}$ is zero. Then the set $\left\{W_{j}\right\}$ on $\mathscr{H}$ is isomorphic with the set $\left\{\alpha_{j} V_{j}\right\}$ on $\mathscr{L}_{2}\left(I_{n}\right)$ where the nonzero real numbers $\alpha_{j}$ are related to $\left\{W_{j}\right\}$ by

$$
\left(W_{j}+W_{j}^{*}\right) e=\alpha_{j} e .
$$

Two sets of operators $\left\{W_{j}\right\}$ as above are unitarily equivalent if and only if they have the same $\alpha$ 's as defined above in (1).

Proof. The proof is based on the following formulas: 


$$
\begin{gathered}
\left(\prod_{j=1}^{n} \alpha_{\jmath}\left(W_{j}-z_{j}\right)^{-1} e, e\right)=\prod_{j=1}^{n}\left(1-\exp \left(\alpha_{j} z_{j}^{-1}\right)\right), \\
\left(\prod_{j=1}^{n} \alpha_{j}\left(Z_{j}-z_{j}\right)^{-1} e, e\right)=\prod_{j=1}^{n}\left(1-\exp \left(\alpha_{\jmath} z_{j}^{-1}\right)\right), \\
\prod_{j=\mathrm{I}}^{n}\left(z_{j 1}+\overline{z_{j 2}}\right)\left(\prod_{j=1}^{n} \alpha_{\jmath}\left(W_{j}-z_{j 1}\right)^{-1} e, \prod_{j=1}^{n} \alpha_{j}\left(W_{j}-z_{j 2}\right)^{-1} e\right) \\
=\prod_{j=1}^{n}\left(\exp \left(\alpha_{j}\left(z_{j 1}^{-1}+\overline{z_{j 2}^{-1}}\right)\right)-1\right),
\end{gathered}
$$

where the $z$ 's are arbitrary nonzero complex numbers and we write $W-z$ instead of $W-z I$ with the identity operator $I$; where $Z_{j}$ is either $W_{j}$ or $W_{j}^{*}$; and where the left side of (2) is the joint characteristic function of the sets $\left\{W_{j}\right\}$ (see [3]). These formulas and the isomorphism of the sets $\left\{W_{j}\right\}$ and $\left\{\alpha_{j} V_{j}\right\}$ will be proved by induction on $n$. The case $n=1$ is again, as in Theorem 1 , the characterization of $\alpha V$ and may be found in [3].

We begin by justifying (1). Define the commuting set of nonzero Hermitian operators $\left\{E_{j}\right\}$ by $E_{j}=W_{j}+W_{j}^{*}$ and set $E=\prod_{j=1}^{n} E_{j}$. Assumption (i) implies that for all $x \in \mathscr{C}$ we have

$$
E x=\alpha(x, e) e
$$

for some real nonzero $\alpha$. This implies that $E_{j} e=\left(E_{j} e, e\right)=\alpha_{j} e$ and so

$$
E_{j_{1}} \cdots E_{j_{s}} e=\alpha_{j_{1}} \cdots \alpha_{j_{s}} e
$$

so that $\alpha=\prod_{j=1}^{n} \alpha_{j}$ and the $\alpha_{j}$ are not zero.

In order to simplify the exposition, we replace the $W_{j}^{\prime}$ 's by $\alpha_{j}^{-1} W_{j}$ and then establish the theorem and the relevant formulas (1)-(3) for the special case where all the $\alpha$ 's are 1 . The results for the original $W_{j}$ 's are then obtained by replacing the $z$ 's by the corresponding $\alpha^{-1} z^{\prime}$ s.

The induction hypothesis uses the conclusions of the theorem and the formulas (1)-(3) for all $j<n$. We first establish (2), then (3), then the isomorphism of the sets $\left\{W_{j}\right\}$ and $\left\{V_{j}\right\}$, and then $\left(2^{\prime}\right)$.

We apply the identity

$$
\begin{aligned}
\left(z_{1}+\bar{z}_{2}\right) & \left(W^{*}-\bar{z}_{2}\right)^{-1}\left(W-z_{1}\right)^{-1} \\
= & -\left(W-z_{1}\right)^{-1}-\left(W^{*}-\bar{z}_{2}\right)^{-1} \\
& \quad+\left(W^{*}-\bar{z}_{2}\right)^{-1}\left(W+W^{*}\right)\left(W-z_{1}\right)^{-1}
\end{aligned}
$$

to the left side $L$ of (3) and obtain

$$
\begin{aligned}
L & \equiv\left(\prod_{j=1}^{n}\left[-\left(W_{j}-z_{j_{1}}\right)^{-1}-\left(W_{j}^{*}-\overline{z_{j 2}}\right)^{-1}+\left(W_{j}^{*}-\overline{z_{j 2}}\right)^{-1} E_{j}\left(W_{j}-z_{j_{1}}\right)^{-1}\right] e, e\right) \\
& =\left(\prod_{j=1}^{n}\left[-A_{j}-B_{j}+B_{j} E_{j} A_{j}\right] e, e\right) \equiv R
\end{aligned}
$$


where $A_{j}=\left(W_{j}-z_{j 1}\right)^{-1}$ and $B_{j}=\left(W_{j}^{*}-\overline{z_{j 2}}\right)^{-1}$. In the expansion of $R$ "we separate out the last factor so that

$$
R=\left(\left[\prod_{j=1}^{n-1}\left(-A_{j}-B_{j}+B_{j} E_{j} A_{j}\right)\right]\left(-A_{n}-B_{n}+B_{n} E_{n} A_{n}\right) e, e\right)
$$

and we write $R=\sum X+Y$ where the terms designated by $X$ are of the form

$$
\begin{aligned}
X= & (-1)^{n-t}\left(\left(\prod_{j=1}^{n-1} C_{j}\right) A_{n} F_{1} \cdots F_{t} D_{1} \cdots D_{t} e, e\right) \\
& +(-1)^{n-t}\left(\left(\prod_{j=1}^{n-1} C_{j}\right) B_{n} F_{1} \cdots F_{t} D_{1} \cdots D_{t} e, e\right) \\
& +(-1)^{n-t+1}\left(\left(\prod_{j=1}^{n-1} C_{j}\right) B_{n} F_{1} \cdots F_{t} E_{n} D_{1} \cdots D_{t} A_{n} e, e\right)
\end{aligned}
$$

where: $C_{j}$ is either $A_{j}$ or $B_{j} ; F_{k}=E_{j(k)}$ and $D_{k}=C_{j(k)}$ for $j(k)$ a suitable permutation of a subset $T$ of $\{1, \cdots, n-1\}$ containing $t \leqq n-2$ elements (or the $F^{\prime}$ 's and $D$ 's are absent and we set $t=0$ ); and where

$$
Y=\left(\left[\prod_{j=1}^{n-1}\left(B_{j} E_{j} A_{j}\right)\right]\left(-A_{n}-B_{n}+B_{n} E_{n} A_{n}\right) e, e\right) .
$$

Now (4) and (5) imply that

$$
F_{1} \cdots F_{t} D_{1} \cdots D_{t} e=E D_{1} \cdots D_{t} e=\left(D_{1} \cdots D_{t} e, e\right) e
$$

and similarly

$$
F_{1} \cdots F_{t} E_{n} D_{1} \cdots D_{t} A_{n} e=\left(D_{1} \cdots D_{t} A_{n} e, e\right) e .
$$

We now wish to use our induction hypothesis in order to calculate the right sides of (7) and (8). Relabel the indices so that $T=\{1, \cdots, t\}$ with $t \leqq n-1$ for our present purpose and set $\mathscr{H}_{t}=E_{n} \cdots E_{t+1} \mathscr{\mathscr { C }}$. Assumption (ii) implies that $\mathscr{H}_{t}$ is invariant under $W_{j}$ and $W_{j}^{*}$ for all $j \in T$ and clearly $e \in \mathscr{H}_{t}$. In $\mathscr{H}_{t}$ we consider the closed linear subspace $\mathscr{K}$ generated by $\left\{W_{j}\right\}_{j \in T}$ and $e$. Clearly $\mathscr{K}$ is invariant under $\left\{W_{j}\right\}_{j \in T}$. We wish to show that this set of operators restricted to $\mathscr{K}$ satisfies the hypotheses of our theorem; it clearly suffices to establish (ii): to that end we show that $\mathscr{C}$ is invariant with respect to all $W_{j}^{*}, j \in T$. We observe that $\mathscr{K}^{\prime}$, the orthogonal complement of $\mathscr{K}$ in $\mathscr{K}_{t}$, is invariant under all $E_{j}$ : take $x^{\prime} \in \mathscr{K}^{\prime}$, then $\left(x^{\prime}, W_{1}^{p_{1}} \ldots W_{t}^{p_{t}} e\right)=0$ for all nonnegative integral exponents $p$ and $\left(E_{j} x^{\prime}, W_{1}^{p_{1}} \cdots W_{t}^{p_{t}} e\right)=$ $\left(x^{\prime}, W_{1}^{p_{1}} \cdots W_{j}^{0} \cdots W_{t}^{p_{t}} E_{j} W_{j}^{p_{j}} e\right)=\left(x^{\prime}, W_{1}^{p_{1}} \cdots W_{j}^{0} \cdots W_{t}^{p_{t}}\right)\left(W_{j}^{p_{j}} e, e\right)=0$ since $E_{j} W_{j}^{p_{j}} e=E W_{j}^{p_{j}} e=\left(W_{j}^{p_{j}} e, e\right) e$ by (4) and (5); we have used the convention $W^{0}=I$, the identity operator. Thus $\mathscr{K}^{\prime}$ and hence $\mathscr{K}$ is invariant under $E_{j}$ and hence $\mathscr{K}$ is invariant under $W_{j}^{*}$ : take $x \in \mathscr{K}$, 
then $W_{j}^{*} x=\left(E_{j}-W_{j}\right) x \in \mathscr{K}$. Thus all our formulas are applicable and we have in particular for the right side of (7)

$$
\left(D_{1} \cdots D_{t} e, e\right)=\prod_{\jmath=1}^{t}\left(1-\exp \left(w_{j}^{-1}\right)\right)=P
$$

where $w_{j}$ is $z_{\jmath 1}$ or $\overline{z_{j 2}}$ depending on whether the corresponding $D_{j}$ is an $A_{j}$ or a $B_{j}$; note that we have made use of $\left(2^{\prime}\right)$. In order to calculate the right side of (8) note that since $t \leqq n-2$, our induction hypothesis is applicable and we obtain

$$
\left(D_{1} \cdots D_{t} A_{n} e, e\right)=\left(1-\exp \left(z_{n 1}^{-1}\right)\right) P .
$$

Hence (7) and (8) imply that

$$
X=(-1)^{n-t} P\left[\left(\left(\prod_{j=1}^{n-1} C_{j}\right) A_{n} e, e\right)+\exp \left(z_{n 1}^{-1}\right)\left(\left(\prod_{j=1}^{n-1} C_{j}\right) B_{n} e, e\right)\right] .
$$

If in (6) we set $-\bar{z}_{2}=z_{1}=z_{j_{1}}$ and $W=W_{j}$, we obtain $\left(W_{j}-z_{j_{1}}\right)^{-1}=$ $\left(W_{j}^{*}+z_{j 1}\right)^{-1} E_{j}\left(W_{j}-z_{j_{1}}\right)^{-1}-\left(W_{j}^{*}+z_{j 1}\right)^{-1}$ so that after a little calculation and using $E_{j} A_{j} e=\left(A_{j} e, e\right) e=\left(1-\exp \left(z_{j 1}^{-1}\right)\right) e$ we have

$$
\begin{aligned}
& \left(D_{1} \cdots D_{j-1} A_{j} D_{j+1} \cdots D_{n} e, e\right) \\
& \quad=-\exp \left(z_{j 1}^{-1}\right)\left(D_{1} \cdots D_{j-1}\left(W_{j}^{*}+z_{j 1}\right)^{-1} D_{j+1} \cdots D_{n} e, e\right) .
\end{aligned}
$$

In a similar way, if we set in (6) $-\bar{z}_{2}=z_{1}=z_{j 2}$ and $W=W_{j}^{*}$, we obtain

$$
\begin{aligned}
& \left(D_{1} \cdots D_{j-1} B_{j} D_{j+1} \cdots B_{n} e, e\right) \\
& \quad=-\exp \left({\overline{z_{j 2}}}^{-1}\right)\left(D_{1} \cdots D_{j-1}\left(W_{j}+{\overline{z_{j 2}}}^{-1} D_{j+1} \cdots D_{n} e, e\right)\right.
\end{aligned}
$$

so that if we set $-\overline{z_{n 2}}=z_{n 1}$, then $X$ is seen to be identically 0 . We now turn to $Y$. A little calculation shows that

$$
Y=-\left(A_{1} \cdots A_{n-1} e, e\right)\left[\left(B_{1} \cdots B_{n-1} A_{n} e, e\right)+\left(B_{1} \cdots B_{n} e, e\right)\right]+a b
$$

where $a=\left(A_{1} \cdots A_{n} e, e\right)$ and $b=\left(B_{1} \cdots B_{n} e, e\right)$. We now use (9), set $-\overline{z_{n 2}}=z_{n 1}$, and use our induction hypothesis to conclude that

$$
Y=b\left[a-\prod_{j=1}^{n-1}\left(1-\exp \left(z_{j 1}^{-1}\right)\right)\left(1-\exp \left(z_{n 1}^{-1}\right)\right)\right] .
$$

Since now $L=R=\sum X+Y$, the substitution $-\overline{z_{n 2}}=z_{n 1}$ implies that $L$ and $R$ are identically zero; $X$ is also identically zero and therefore $Y$ must be identically zero and therefore $Y$ must be identically zero. Since, however, $b$ is not identically zero, we can conclude that $a-\prod_{j=1}^{n}\left(1-\exp \left(z_{j 1}^{-1}\right)\right)$ must be identically zero. Thus (2) is established.

To prove (3), we turn once again to $L=R=\sum X+Y$; $\left(9^{\prime}\right)$ shows that in the expansion of $R$ we can successively replace $B$ 's by $A$ 's 
(and exponentials), so that finally $L$ can be expressed entirely in terms of exponentials and terms like the right side $R^{\prime}$ of (2). Now the set $\left\{V_{j}\right\}$ on $\mathscr{L}_{2}\left(I_{n}\right)$ satisfies the hypotheses of our theorem (all the $\alpha$ 's still being 1); therefore (2) is true if we replace $\left\{W_{j}\right\}$ by $\left\{V_{j}\right\}$. The left side of (3) equals $\sum X+Y$; these terms can be expressed entirely in terms of $A$ 's and other exponentials. The way these $A$ 's and other exponentials occur is based only on the hypotheses (i)-(iii) of the theorem which are satisfied by $\left\{W_{j}\right\}$ as well as $\left\{V_{j}\right\}$. Hence, since the $A$ 's are the same for these two sets, i.e., since (2) with all the $\alpha$ 's equal to 1 is true for both sets, the left side of (3) (with the $\alpha$ 's equal to 1) is also the same for both sets. Thus if we calculate $L$ for $\left\{V_{j}\right\}$, we must get same thing as if we do it for $\left\{W_{j}\right\}$; the former calculation is elementary and yields the desired right side of (3) which is thus established for $\left\{W_{j}\right\}$.

In order to prove the isomorphism of the sets $\left\{W_{j}\right\}$ and $\left\{V_{j}\right\}$ we observe that (3) is valid and equal for both sets. Thus the infinite power series expansion deduced from (3) implies that for all nonnegative exponents, we have

$$
\left(W_{1}^{p_{11}} \cdots W_{n}^{p_{n 1}} e, W_{1}^{p_{12}} \cdots W_{n}^{p_{n 2} e} e\right)=\left(V_{1}^{p_{11}} \cdots V_{n}^{p_{n 1}} e, V_{1}^{p_{12}} \cdots V_{n}^{p_{n 2}} e\right) .
$$

Since $e$ and $\left\{W_{j}\right\}$ generate $\mathscr{H}$ and the function identically equal to 1 and the set $\left\{V_{j}\right\}$ generate $\mathscr{L}_{2}\left(I_{n}\right)$, we can apply Livšic's Lemma and conclude the desired isomorphism.

The last step is to check $\left(2^{\prime}\right)$. In view of the isomorphism of $\left\{W_{j}\right\}$ and $\left\{V_{j}\right\}$, it suffices to verify it for this latter set. If $u$ is the function identically equal to 1 , it is easy to verify by induction that

$$
\prod_{j=1}^{k}\left(V_{j}-z_{j}\right)^{-1} u=(-1)^{k} \prod_{j=1}^{k} z_{j}^{-1} \exp \left(z_{j}^{-1} x_{j}\right) .
$$

We now rewrite the left side of $\left(2^{\prime}\right)$ and obtain after eventual relabelling of indices and still keeping the $\alpha$ 's equal to 1 the expression $\left(\prod_{j=1}^{k}\left(V_{j}-z_{j}\right)^{-1} u, \Pi_{j=k+1}^{n}\left(V_{j}-\bar{z}_{j}\right)^{-1} u\right)$. A simple calculation then shows that this equals the right side of $\left(2^{\prime}\right)$; in view of the preceding paragraph $u$ and $e$ are identified.

Just as was the case in Theorem 1 , the fact that the set $\left\{\alpha_{j}\right\}$ determines $\left\{W_{j}\right\}$ unitarily is an immediate consequence of our representation. This completes the proof of the theorem.

\section{REFERENCES}

1. M. S. Brodskiǐ and M. S. Livšic, Spektral'nyı̃ analiz nesamosopražennyh operatorov $i$ promežutočnye sistemy, Usp. mat. nauk 13, \#1, (1958), 3-85, = Amer. Math. Soc. Transl. (2), 13 (1960), 265-346 (Spectral analysis of non-selfadjoint operators and intermediate systems). 
2. G. K. Kalisch, On similarity, reducing manifolds, and unitary equivalence of certain Volterra operators, Ann. of Math. 66 (1957), 481-494.

3. G. K. Kalisch, Direct proofs of spectral representation theorems, Journal of Math. Anal. and Appl. 8 (1964), 351-363.

4. M. S. Livšic, O spelttral'nom razloženii liněnyh nesamosopražennyh operatorov, Mat. Sb. 34 (1954), 145-198=Amer. Math. Soc. Transl. (2), 5 (1957), 67-114 (On spectral decomposition of linear non-selfadjoint operators).

Received March 5, 1964, and in revised form December 31, 1964. The author was supported in part by grant NSFG 14137 from the National Science Foundation, herewith gratefully acknowledged.

UNIVERSITY OF MINNESOTA 


\section{PACIFIC JOURNAL OF MATHEMATICS}

\section{EDITORS}

\author{
H. SAMELSON, \\ Stanford University \\ Stanford, California \\ R. M. BLUMENTHAL \\ University of Washington \\ Seattle, Washington 98105
}

\author{
*J. DugundJI \\ University of Southern California \\ Los Angeles, California 90007 \\ RICHARD ARENS \\ University of California \\ Los Angeles, California 90024
}

\section{ASSOCIATE EDITORS}
E. F. BECKENBACH
B. H. NeUmanN
F. WOLF
K. YOSIDA

\section{SUPPORTING INSTITUTIONS}

\author{
UNIVERSITY OF BRITISH COLUMBIA \\ CALIFORNIA INSTITUTE OF TECHNOLOGY \\ UNIVERSITY OF CALIFORNIA \\ MONTANA STATE UNIVERSITY \\ UNIVERSITY OF NEVADA \\ NEW MEXICO STATE UNIVERSITY \\ OREGON STATE UNIVERSITY \\ UNIVERSITY OF OREGON \\ OSAKA UNIVERSITY \\ UNIVERSITY OF SOUTHERN CALIFORNIA
}

\author{
STANFORD UNIVERSITY \\ UNIVERSITY OF TOKYO \\ UNIVERSITY OF UTAH \\ WASHINGTON STATE UNIVERSITY \\ UNIVERSITY OF WASHINGTON \\ * * * \\ AMERICAN MATHEMATICAL SOCIETY \\ CHEVRON RESEARCH CORPORATION \\ TRW SYSTEMS \\ NAVAL ORDNANCE TEST STATION
}

Mathematical papers intended for publication in the Pacific Journal of Mathematics should be typewritten (double spaced). The first paragraph or two must be capable of being used separately as a synopsis of the entire paper. It should not contain references to the bibliography. Manuscripts may be sent to any one of the four editors. All other communications to the editors should be addressed to the managing editor, Richard Arens at the University of California, Los Angeles, California 90024 .

50 reprints per author of each article are furnished free of charge; additional copies may be obtained at cost in multiples of 50 .

The Pacific Journal of Mathematics is published monthly. Effective with Volume 16 the price per volume (3 numbers) is $\$ 8.00$; single issues, $\$ 3.00$. Special price for current issues to individual faculty members of supporting institutions and to individual members of the American Mathematical Society: $\$ 4.00$ per volume; single issues $\$ 1.50$. Back numbers are available.

Subscriptions, orders for back numbers, and changes of address should be sent to Pacific Journal of Mathematics, 103 Highland Boulevard, Berkeley 8, California.

Printed at Kokusai Bunken Insatsusha (International Academic Printing Co., Ltd.), No. 6, 2-chome, Fujimi-cho, Chiyoda-ku, Tokyo, Japan.

PUBLISHED BY PACIFIC JOURNAL OF MATHEMATICS, A NON-PROFIT CORPORATION

The Supporting Institutions listed above contribute to the cost of publication of this Journal, but they are not owners or publishers and have no responsibility for its content or policies.

* Paul A. White, Acting Editor until J. Dugundji returns. 


\section{Pacific Journal of Mathematics}

\section{Vol. 18, No. 3 \\ May, 1966}

William George Bade and Philip C. Curtis, Jr., Embedding theorems for commutative Banach algebras .......................... 391

Wilfred Eaton Barnes, On the $\Gamma$-rings of Nobusawa ................. 411

J. D. Brooks, Second order dissipative operators ................ 423

Selwyn Ross Caradus, Operators with finite ascent and descent ........ 437

Earl A. Coddington and Anton Zettl, Hermitian and anti-hermitian properties of Green's matrices .......................... 451

Robert Arnold Di Paola, On sets represented by the same formula in distinct consistent axiomatizable Rosser theories ................... 455

Mary Rodriguez Embry, Conditions implying normality in Hilbert space ...........................................

Garth Ian Gaudry, Quasimeasures and operators commuting with convolution ................................... 461

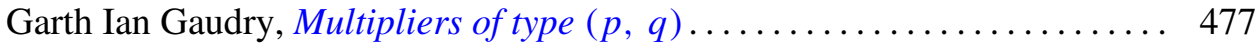

Ernest Lyle Griffin, Jr., Everywhere defined linear transformations affiliated with rings of operators .................................. 489

Philip Hartman, On the bounded slope condition ................ 495

David Wilson Henderson, Relative general position ................ 513

William Branham Jones, Duality and types of completeness in locally

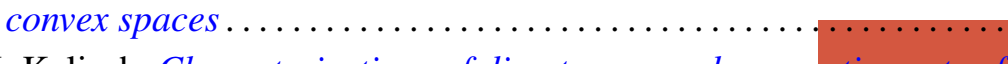

G. K. Kalisch, Characterizations of direct sums and commuting sets of Volterra operators....

Ottmar Loos, Über eine Beziehung zwischen Malcev-Algebren und Lietripelsystemen

Ronson Joseph Warne, A class of bisimple inverse semigroups . . . 